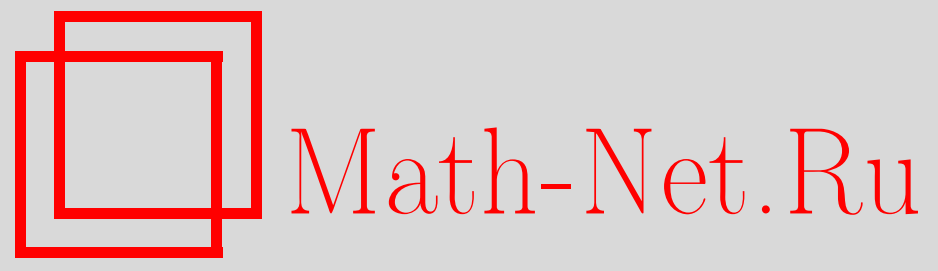

Ю. Л. Павлов, Е. В. Черепанова, Предельное распределение числа пар в обобщенной схеме размещения, Дискрет. матем., 2002, том 14, выпуск 3, 149-159

DOI: https://doi.org/10.4213/dm262

Использование Общероссийского математического портала Math-Net.Ru подразумевает, что вы прочитали и согласны с пользовательским соглашением http://www . mathnet.ru/rus/agreement

Параметры загрузки:

IP : 35.173 .137 .237

26 апреля 2023 г., 15:56:34

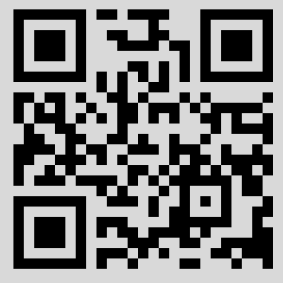


Удк 519.2

\title{
Предельное распределение числа пар в обобщенной схеме размещения
}

\author{
( 2002 г. Ю. Л. Павлов, Е. В. Черепанова
}

\begin{abstract}
Рассматриваются три комбинаторные задачи, описываемые обобщенной схемой размещения: извлечение шаров из урны, содержащей шары разных цветов, случайные леса и случайные подстановки с известным числом циклов. В этих задачах получены предельные распределения числа пар шаров одного цвета, числа цепей леса и числа простых путей в графе подстановки соответственно.
\end{abstract}

Пусть имеется последовательность $n$ независимых испытаний с $N$ исходами, $p_{i}-$ вероятность $i$-го исхода в каждом испытании, $1 \leqslant i \leqslant N$. В работе В. Ф. Колчина [1] получена локальная предельная теорема для случайной величины

$$
\eta=\frac{1}{2} \sum_{i=1}^{N} v^{(i)}\left(v^{(i)}-1\right),
$$

где $v^{(i)}$ - число появлений $i$-го исхода, $1 \leqslant i \leqslant N$, в случае, когда $n, N \rightarrow \infty$, $0<c_{0} \leqslant N p_{i} \leqslant c_{1}<\infty, 1 \leqslant i \leqslant N, n^{2} / N \rightarrow \infty, N^{4} /\left(n^{3} \ln n\right) \rightarrow \infty$. Заметим, что проблема получения предельных теорем для сумм вида (1) возникает в различных комбинаторных задачах, отличающихся от указанной полиномиальной схемы. Некоторые из таких случаев приводятся ниже.

В [2] рассматривалась следующая задача. Из урны, содержащей по $m \geqslant 2$ различных шаров каждого из $N$ цветов, осуществляется равновероятный выбор без возвращения $n$ шаров, $0<n<m N$. Требуется найти предельное распределение случайной величины $\nu_{1}$, равной числу всевозможных пар извлеченных шаров одинакового цвета, при $n, N \rightarrow \infty$. В [2] сформулирована локальная предельная теорема для $v_{1}$ в случае, когда $m$ фиксировано и $0<\alpha_{0} \leqslant n /(m N) \leqslant \alpha_{1}<1$.

Для доказательства данной теоремы предложено использовать обобщенную схему размещения частиц по ячейкам, введенную и исследованную в книгах В. Ф. Колчина $[3,4,5]$. В этой схеме предполагается, что набор целочисленных случайных величин $\eta_{1}, \ldots, \eta_{N}$ таких, что

$$
\eta_{1}+\ldots+\eta_{N}=n
$$


связан с независимыми одинаково распределенными целочисленными случайными величинами $\xi_{1}, \ldots, \xi_{N}$ соотношением

$$
\mathbf{P}\left\{\eta_{1}=k_{1}, \ldots, \eta_{N}=k_{N}\right\}=\mathbf{P}\left\{\xi_{1}=k_{1}, \ldots, \xi_{N}=k_{N} \mid \xi_{1}+\ldots+\xi_{N}=n\right\} .
$$

В рассматриваемой задаче $\eta_{i}$ является случайной величиной, равной числу извлеченных шаров $i$-го цвета, $1 \leqslant i \leqslant N$, а $\xi_{1}, \ldots, \xi_{N}$ распределены по биномиальному закону с параметрами $m$ и $p, 0<p<1$. Очевидно, что данную задачу можно рассматривать как частный случай проблемы получения предельных распределений числа пар $v$ в обобщенной схеме размещения, где

$$
v=\eta_{1}\left(\eta_{1}-1\right) / 2+\ldots+\eta_{N}\left(\eta_{N}-1\right) / 2 .
$$

Из (2) следует, что

$$
\begin{aligned}
\mathbf{P}\{v=k\} & =\mathbf{P}\left\{\zeta_{N}=k \mid \mu_{N}=n\right\} \\
& =\frac{\mathbf{P}\left\{\zeta_{N}=k, \mu_{N}=n\right\}}{\mathbf{P}\left\{\mu_{N}=n\right\}},
\end{aligned}
$$

где

$$
\begin{aligned}
\zeta_{N} & =\xi_{1}\left(\xi_{1}-1\right) / 2+\ldots+\xi_{N}\left(\xi_{N}-1\right) / 2 \\
\mu_{N} & =\xi_{1}+\ldots+\xi_{N} .
\end{aligned}
$$

Нетрудно видеть, что суммы случайных величин в (3) образуют схемы серий. Поскольку классические локальные предельные теоремы в этом случае неприменимы (см. по этому поводу замечание в книге [4]), задача получения предельного распределения $v$ сводится к доказательству локальных предельных теорем для $\mu_{N}$ и вектора $\left(\zeta_{N}, \mu_{N}\right)$. Заметим, что в [2] такие доказательства не приводятся.

В данной работе получено обобщение указанного выше результата для более широкой области изменения параметров, а также доказаны предельные теоремы для числа пар объектов в случайных лесах и подстановках с заданным числом циклов (см. $[3,4,5])$.

Теорема 1. Пусть $m \geqslant 2,0<n<m N, n, N \rightarrow \infty$ так, что $m N-n \rightarrow \infty$ и выполняется одно из следующих условий:

(1) $n / N \rightarrow 0, n^{2} / N \rightarrow \infty$;

(2) $0<a_{1} \leqslant n / N \leqslant a_{2}<\infty, n(1-n /(m N))^{3} \rightarrow \infty$;

(3) $n / N \rightarrow \infty,(1-n /(m N)) N^{4 / 3} / n \rightarrow \infty,(1-n /(m N)) n^{3} / N^{3}=O(1)$.

Тогда

$$
\mathbf{P}\left\{\nu_{1}=k\right\}=\frac{1}{\sigma \sqrt{2 \pi}} \exp \left\{-\frac{(k-a)^{2}}{2 \sigma^{2}}\right\}(1+o(1))
$$

равномерно относительно $k$, для которых $(k-a) / \sigma$ лежит в любом фиксированном конечном интервале, где

$$
\begin{aligned}
a & =\frac{(m-1) n^{2}}{2 m N} \\
\sigma^{2} & =\frac{(m-1)(m N-n)^{2} n^{2}}{2 m^{3} N^{3}} .
\end{aligned}
$$


Пусть $F_{N, n}-$ множество всех различных лесов с $N$ корневыми и $n$ некорневыми вершинами, в которых корни занумерованы числами от 1 до $N$, а некорневые вершины числами от 1 до $n$. Зададим на $F_{N, n}$ равномерное распределение вероятностей. Случайные леса такого типа изучались ранее в [6, 7]. В [7] показано, что равенство (2) справедливо, если $\eta_{i}$ равно числу некорневых вершин дерева с корнем, имеющим номер $i, 1 \leqslant i \leqslant N$, случайные величины $\xi_{1}, \ldots, \xi_{N}$ таковы, что

$$
\mathbf{P}\left\{\xi_{1}=k\right\}=\frac{(k+1)^{k} x^{k+1}}{(k+1) ! \theta(x)}, \quad k=0,1, \ldots,
$$

где $x$ - числовой параметр, $0<x \leqslant e^{-1}$, а функция $\theta(x)$ определяется рядом

$$
\theta(x)=\sum_{k=1}^{\infty} \frac{k^{k-1}}{k !} x^{k}
$$

Под парой будем понимать две некорневые вершины, соединенные цепью. Обозначим через $v_{2}$ случайную величину, равную числу всевозможных пар вершин в лесе из $F_{N, n}$. Легко видеть, что число различных цепей в лесе равно

$$
\tau=v_{2}+n
$$

Следующая теорема устанавливает предельное распределение числа цепей в случайном лесе.

Теорема 2. Пусть $n, N \rightarrow \infty$ так, что $n / N=O(1), n^{2} / N \rightarrow \infty$. Тогда

$$
\mathbf{P}\{\tau-n=k\}=\frac{1}{\sigma \sqrt{2 \pi}} \exp \left\{-\frac{(k-a)^{2}}{2 \sigma^{2}}\right\}(1+o(1))
$$

равномерно относительно $k$, для которых $(k-a) / \sigma$ лежит в любом фиксированном конечном интервале, где

$$
\begin{aligned}
a & =\frac{n^{2}(n+3 N)}{2 N^{2}}, \\
\sigma^{2} & =\frac{3 n^{2}(n+N)^{5}}{2 N^{6}} .
\end{aligned}
$$

Рассмотрим множество $S_{n, N}$ всех различных подстановок степени $n$, имеющих $N$ циклов. Легко видеть, что подстановку можно представить ориентированным графом, каждая компонента связности которого является циклом. Введем на $S_{n, N}$ равномерное распределение вероятностей. Известно [4], что соотношение (2) выполняется, если случайная величина $\eta_{i}$ равна числу элементов в $i$-ом цикле, $1 \leqslant i \leqslant N$, а $\xi_{1}, \ldots, \xi_{N}$ имеют распределение логарифмического ряда

$$
\mathbf{P}\left\{\xi_{1}=k\right\}=\frac{\lambda^{k}}{-k \ln (1-\lambda)}, \quad k=1,2, \ldots,
$$

где $\lambda$ - числовой параметр, $0<\lambda<1$. Будем считать, что два различных элемента подстановки образуют пару, если они принадлежат одному циклу. Пусть $\nu_{3}$ - случайная 
величина, равная числу различных пар элементов подстановки из $S_{n, N}$. Нетрудно видеть, что случайная величина $2 \nu_{3}$ равна числу простых (незамкнутых) путей графа подстановки.

Теорема 3. Пусть $\lambda$-решение уравнения

$$
\frac{\lambda}{(\lambda-1) \ln (1-\lambda)}=\frac{n}{N}
$$

в интервале $(0,1)$ и пусть $n, N \rightarrow \infty$ так, что $1<c_{1} \leqslant n / N \leqslant c_{2}<\infty$. Тогда

$$
\mathbf{P}\left\{\nu_{3}=k\right\}=\frac{1}{\sigma \sqrt{2 \pi}} \exp \left\{-\frac{(k-a)^{2}}{2 \sigma^{2}}\right\}(1+o(1))
$$

равномерно относительно $k$, для которых $(k-a) / \sigma$ лежит в любом фиксированном конечном интервале, где

$$
\begin{aligned}
a & =\frac{n \lambda}{2(1-\lambda)} \\
\sigma^{2} & =\frac{n \lambda}{4(1-\lambda)^{3}}\left(\frac{\lambda^{2}}{\ln (1-\lambda)+\lambda}+2\right) .
\end{aligned}
$$

Ниже приводятся вспомогательные утверждения (леммы 1-8), с помощью которых далее будут доказаны теоремы 1-3.

Пусть $\langle\alpha\rangle$ обозначает расстояние от $\alpha \in \mathbf{R}$ до ближайшего целого числа,

$$
\Omega(\alpha, \beta)=\left\{d=\left(d_{1}, d_{2}\right) \in \mathbf{R}^{2}|| d_{1}|,| d_{2}|\leqslant \alpha,| d \mid>\beta\right\} .
$$

Для двумерной случайной величины $X$ и вектора $d \in \mathbf{R}^{2}$ положим

$$
H_{N}(X, d)=\mathbf{M}\left\langle\left(X^{*}, d\right)\right\rangle^{2},
$$

где $\left(X^{*}, d\right)$ - скалярное произведение векторов $X^{*}$ и $d, X^{*}$ получен из $X$ путем симметризации. Введем обозначения

$$
\begin{gathered}
\tau_{i}=\xi_{i}\left(\xi_{i}-1\right) / 2, \quad X_{i}=\left(\tau_{i}, \xi_{i}\right), \quad 1 \leqslant i \leqslant N, \\
S_{N}=X_{1}+\ldots+X_{N}=\left(\zeta_{N}, \mu_{N}\right), \\
\sigma_{1}^{2}=\mathrm{D} \tau_{1}, \\
\rho=\operatorname{cov}\left(\tau_{1}, \xi_{1}\right) /\left(\sigma_{1} \sigma_{2}\right), \quad \begin{array}{c}
\sigma_{2}^{2}=\mathrm{D} \xi_{1}, \\
A_{N}=\mathbf{M} S_{N},
\end{array} \\
\Sigma=\frac{1}{1-\rho^{2}}\left(\begin{array}{cc}
1 & -\rho \\
-\rho & 1
\end{array}\right), \quad Q_{N}=\left(\begin{array}{cc}
1 /\left(\sigma_{1} \sqrt{N}\right) & 0 \\
0 & 1 /\left(\sigma_{2} \sqrt{N}\right)
\end{array}\right), \\
\left\|Q_{N}\right\| \equiv \sup _{|x|=1}\left|Q_{N} x\right|, \quad \\
H_{N}(d)=N H\left(X_{1}, d\right), \quad H_{N}=\inf _{d \in \Omega(1 / 2,1 / 4)} H_{N}(d) .
\end{gathered}
$$

Несложно показать, что

$$
H_{N}(d)=N \sum_{k, l}\left\langle\alpha_{k, l}\right\rangle^{2} \mathbf{P}\left\{\xi_{1}=k\right\} \mathbf{P}\left\{\xi_{2}=l\right\}
$$


где

$$
\alpha_{k, l}=(k-l)(k+l-1) d_{1} / 2+(k-l) d_{2} .
$$

Заметим, что

$$
\left(S_{N}-A_{N}\right) Q_{N}=\left(\frac{\zeta_{N}-\mathbf{M} \zeta_{N}}{\sigma_{1} \sqrt{N}}, \frac{\mu_{N}-\mathbf{M} \mu_{N}}{\sigma_{2} \sqrt{N}}\right) .
$$

Согласно теореме 4 в [8] имеет место следующее утверждение.

Лемма 1. Пусть при $N \rightarrow \infty$ последовательность распределений случайных величин $\left(S_{N}-A_{N}\right) Q_{N}$ слабо сходится к абсолютно непрерывному распределению. Тогда для локальной сходимости распределений $S_{N}$ к тому же распределению достаточно, чтобы выполнялось условие

$$
\left\|Q_{N}^{-1}\right\|^{2}=O\left(H_{N}\right)
$$

Лемма 2. Для любого $d \in \Omega(1 / 2,1 / 4)$ и любых чельх $k, l$ найдется такое $\varepsilon>0$, что

$$
\max \left\{\left\langle\alpha_{k, l}\right\rangle,\left\langle\alpha_{k+1, l}\right\rangle,\left\langle\alpha_{k+2, l}\right\rangle\right\} \geqslant \varepsilon \text {. }
$$

Доказательство. Покажем, что утверждение леммы справедливо при

$$
\varepsilon= \begin{cases}1 / 8, & \text { если } d_{1}=0 \\ \left|d_{1}\right| / 4, & \text { если } d_{1} \neq 0\end{cases}
$$

Легко проверить, что

$$
\begin{aligned}
\alpha_{k+1, l} & =\alpha_{k, l}+k d_{1}+d_{2}, \\
\alpha_{k+2, l} & =\alpha_{k, l}+2\left(k d_{1}+d_{2}\right)+d_{1} .
\end{aligned}
$$

Представим $\alpha_{k, l}$ и $k d_{1}+d_{2}$ в виде сумм

$$
\begin{aligned}
\alpha_{k, l} & =z_{1}+r_{1}, \\
k d_{1}+d_{2} & =z_{2}+r_{2},
\end{aligned}
$$

где $z_{1}, z_{2} \in \mathbf{Z}, r_{1}, r_{2} \in \mathbf{R},\left|r_{1}\right|,\left|r_{2}\right| \leqslant 1 / 2$. Тогда

$$
\begin{aligned}
\left\langle\alpha_{k, l}\right\rangle & =\left|r_{1}\right|, \\
\left\langle\alpha_{k+1, l}\right\rangle & =\left\langle r_{1}+r_{2}\right\rangle, \\
\left\langle\alpha_{k+2, l}\right\rangle & =\left\langle r_{1}+2 r_{2}+d_{1}\right\rangle .
\end{aligned}
$$

Ясно, что утверждение леммы справедливо, если $\left|r_{1}\right| \geqslant \varepsilon$. Пусть $\left|r_{1}\right|<\varepsilon$. Рассмотрим векторы вида $d=\left(0, d_{2}\right)$. По определению множества $\Omega(1 / 2,1 / 4)$ в этом случае

$$
1 / 4<\left|d_{2}\right| \leqslant 1 / 2
$$

а из (8) следует, что

$$
\left\langle\alpha_{k+1, l}\right\rangle=\left\langle r_{1}+d_{2}\right\rangle \text {. }
$$


Пусть

$$
1 / 4<d_{2} \leqslant 1 / 2, \quad 0 \leqslant r_{1}<1 / 8 .
$$

Тогда

$$
1 / 4<r_{1}+d_{2}<5 / 8
$$

и, очевидно,

$$
\left\langle\alpha_{k+1, l}\right\rangle>1 / 8
$$

Для других возможных значений $r_{1}$ и $d_{2}$ это неравенство устанавливается аналогично. Предположим теперь, что $d_{1} \neq 0$, и пусть

$$
0<d_{1} \leqslant 1 / 2, \quad 0 \leqslant r_{1}<d_{1} / 4, \quad 0 \leqslant r_{2} \leqslant 1 / 2 .
$$

Тогда

$$
0 \leqslant r_{1}+r_{2}<d_{1} / 4+1 / 2
$$

Используя (8), несложно показать, что если

$$
d_{1} / 4 \leqslant r_{1}+r_{2}<d_{1} / 4+1 / 2,
$$

To

$$
\left\langle\alpha_{k+1, l}\right\rangle \geqslant d_{1} / 4 .
$$

Если

$$
0 \leqslant r_{1}+r_{2}<d_{1} / 4
$$

To

$$
0 \leqslant r_{2}<d_{1} / 4
$$

и

$$
d_{1} \leqslant r_{1}+2 r_{2}+d_{1}<3 d_{1} / 2
$$

поэтому из (8) получаем, что

$$
\left\langle\alpha_{k+2, l}\right\rangle>d_{1} / 4
$$

Повторяя подобные рассуждения для других значений $r_{1}, r_{2}$ и $d_{1}$ приходим к утверждению леммы 2.

Положим

$$
g(x)=\frac{1}{2 \pi \sqrt{1-\rho^{2}}} \exp \left\{-\frac{1}{2} x \Sigma x^{T}\right\}
$$

где $x=\left(x_{1}, x_{2}\right) \in \mathbf{R}^{2}$. 
Лемма 3. Пусть $\xi_{1}, \ldots, \xi_{N}$ имеют биномиальное распределение с параметрами $m \geqslant 2$ и $p=n /(m N), 0<n<m N, n, N \rightarrow \infty$ так, что выполняется одно из следуюших условий:

(1) $n / N \rightarrow 0, n^{2} / N \rightarrow \infty$

(2) $0<a_{1} \leqslant n / N \leqslant a_{2}<\infty, n(1-n /(m N))^{3} \rightarrow \infty$;

(3) $n / N \rightarrow \infty,(1-n /(m N)) N^{4 / 3} / n \rightarrow \infty$.

Тогда последовательность распределений $\left(S_{N}-A_{N}\right) Q_{N}$ слабо сходится к двумерному нормальному закону с плотностью (9).

Доказательство. Легко проверить, что

$$
\begin{aligned}
\mathbf{M} \xi_{1}= & m p \\
\mathbf{M} \xi_{1}^{3}= & m p\left((m-1)(m-2) p^{2}+3(m-1) p+1\right) \\
\mathbf{D} \xi_{1}= & m p(1-p) \\
\mathbf{M} \tau_{1}= & m(m-1) p^{2} / 2 \\
\mathbf{M} \tau_{1}^{3}= & m(m-1) p^{2}\left((m-2)(m-3)(m-4)(m-5) p^{4}\right. \\
& \quad+12(m-2)(m-3)(m-4) p^{3} \\
& \left.\quad+38(m-2)(m-3) p^{2}+32(m-2) p+4\right) / 8 \\
\mathbf{D} \tau_{1}= & m(m-1) p^{2}(1-p)(2 m p-3 p+1) / 2, \\
\rho= & \sqrt{2(m-1) p /(2 m p-3 p+1)} .
\end{aligned}
$$

Обозначим $f\left(t_{1}, t_{2}\right)$ характеристическую функцию случайного вектора

$$
\left(\tau_{1}-\mathbf{M} \tau_{1}, \xi_{1}-\mathbf{M} \xi_{1}\right) .
$$

При достаточно малых $t_{1}, t_{2}$ справедливо соотношение

$$
f\left(t_{1}, t_{2}\right)=1-t_{1}^{2} \sigma_{1}^{2} / 2-t_{2}^{2} \sigma_{2}^{2} / 2-t_{1} t_{2} \operatorname{cov}\left(\tau_{1}, \xi_{1}\right)+r\left(t_{1}, t_{2}\right),
$$

где

$$
\left|r\left(t_{1}, t_{2}\right)\right| \leqslant \frac{1}{6} \mathbf{M}\left(\left|t_{1}\right|\left|\tau_{1}-\mathbf{M} \tau_{1}\right|+\left|t_{2}\right|\left|\xi_{1}-\mathbf{M} \xi_{1}\right|\right)^{3} .
$$

Поскольку, как нетрудно проверить,

$$
(x+y)^{3} \leqslant 8\left(x^{3}+y^{3}\right)
$$

для всех $x, y \geqslant 0$, находим, что

$$
\left|r\left(t_{1}, t_{2}\right)\right| \leqslant c\left(\left|t_{1}\right|^{3}\left(\mathbf{M} \tau_{1}^{3}+\mathbf{M}^{3} \tau_{1}\right)+\left|t_{2}\right|^{3}\left(\mathbf{M} \xi_{1}^{3}+\mathbf{M}^{3} \xi_{1}\right)\right),
$$

где $c>0$.

Пусть $\varphi\left(t_{1}, t_{2}\right)$ означает характеристическую функцию случайной величины

$$
\left(S_{N}-A_{N}\right) Q_{N}
$$


Легко видеть, что при выполнении условий леммы $\sigma_{1}^{2} N, \sigma_{2}^{2} N \rightarrow \infty$. Поэтому из равенств

$$
\varphi\left(t_{1}, t_{2}\right)=f^{N}\left(t_{1} /\left(\sigma_{1} \sqrt{N}\right), t_{2} /\left(\sigma_{2} \sqrt{N}\right)\right)
$$

и (11) получаем, что

$$
\ln \varphi\left(t_{1}, t_{2}\right)=N \ln \left(1-\frac{t_{1}^{2}}{2 N}-\frac{t_{2}^{2}}{2 N}-\frac{t_{1} t_{2} \rho}{N}+r\left(\frac{t_{1}}{\sigma_{1} \sqrt{N}}, \frac{t_{2}}{\sigma_{2} \sqrt{N}}\right)\right) .
$$

Из (12) следует неравенство

$$
\left|r\left(\frac{t_{1}}{\sigma_{1} \sqrt{N}}, \frac{t_{2}}{\sigma_{2} \sqrt{N}}\right)\right| \leqslant c\left(\left|t_{1}\right|^{3} \frac{\mathbf{M} \tau_{1}^{3}+\mathbf{M}^{3} \tau_{1}}{\left(\sigma_{1} \sqrt{N}\right)^{3}}+\left|t_{2}\right|^{3} \frac{\mathbf{M} \xi_{1}^{3}+\mathbf{M}^{3} \xi_{1}}{\left(\sigma_{2} \sqrt{N}\right)^{3}}\right) .
$$

Используя (10), нетрудно получить оценки величин

$$
\begin{aligned}
& A=\frac{\mathbf{M} \tau_{1}^{3}+\mathbf{M}^{3} \tau_{1}}{\left(\sigma_{1} \sqrt{N}\right)^{3}}, \\
& B=\frac{\mathbf{M} \xi_{1}^{3}+\mathbf{M}^{3} \xi_{1}}{\left(\sigma_{2} \sqrt{N}\right)^{3}}
\end{aligned}
$$

при различных соотношениях между $n$ и $N$ :

$$
\begin{aligned}
A & =O(1 / n \sqrt{N}), \quad B=O(1 /(N \sqrt{n})) & & \text { при } n / N \rightarrow 0 ; \\
A, B & =O\left(\left(N^{2} n(1-n /(m N))^{3}\right)^{-1 / 2}\right) & & \text { при } 0<a_{1} \leqslant n / N \leqslant a_{2}<\infty ; \\
A, B & =O\left(\left(n /(1-n /(m N)) N^{2}\right)^{3 / 2}\right) & & \text { при } n / N \rightarrow \infty .
\end{aligned}
$$

Отсюда следует, что при выполнении условий леммы

$$
r\left(\frac{t_{1}}{\sigma_{1} \sqrt{N}}, \frac{t_{2}}{\sigma_{2} \sqrt{N}}\right)=o\left(\frac{1}{N}\right) .
$$

В силу (13), (14) при $n, N \rightarrow \infty$

$$
\ln \varphi\left(t_{1}, t_{2}\right)=-t_{1}^{2} / 2-t_{2}^{2} / 2-t_{1} t_{2} \rho+o(1)
$$

откуда получаем, что

$$
\varphi\left(t_{1}, t_{2}\right)=\exp \left\{-\left(t_{1}^{2}+t_{2}^{2}+2 t_{1} t_{2} \rho\right) / 2\right\}(1+o(1))
$$

в каждой точке $\left(t_{1}, t_{2}\right)$. Лемма доказана.

Лемма 4. Пусть $\xi_{1}, \ldots, \xi_{N}$ имеют биномиальное распределение с параметрами $m \geqslant 2 u$ $p=n /(m N), 0<n<m N, n, N \rightarrow \infty$ так, что выполняется одно из следующих условий:

(1) $n / N \rightarrow 0, n^{2} / N \rightarrow \infty$ 
(2) $0<a_{1} \leqslant n / N \leqslant a_{2}<\infty, n(1-n /(m N))^{3} \rightarrow \infty$;

(3) $n / N \rightarrow \infty,(1-n /(m N)) N^{4 / 3} / n \rightarrow \infty,(1-n /(m N)) n^{3} / N^{3}=O(1)$.

Тогда

$$
\mathbf{P}\left\{S_{n}=z\right\}=\operatorname{det} Q_{N}\left(g\left(\left(z-A_{N}\right) Q_{N}\right)+o(1)\right)
$$

равномерно по $z=(k, l)$, где $k, l$ - челые неотричательные числа.

Доказательство. На основании лемм 1 и 3 достаточно показать, что

$$
\left\|Q_{N}^{-1}\right\|^{2}=O\left(H_{N}\right)
$$

Пусть $d \in \Omega(1 / 2,1 / 4)$. Используя (7), лемму 2 и свойства биномиальных вероятностей, нетрудно проверить, что

$$
H_{N}(d) \geqslant c N
$$

где $c$ - положительная постоянная, зависящая от $d$. Следовательно, для некоторого $c_{1}>0$ имеет место неравенство

$$
H_{N} \geqslant c_{1} N
$$

Поскольку

$$
\left\|Q_{N}^{-1}\right\|^{2}=\sup _{|x|=1}\left(\sigma_{1}^{2} N x_{1}^{2}+\sigma_{2}^{2} N x_{2}^{2}\right)
$$

используя (10), получаем, что

$$
\left\|Q_{N}^{-1}\right\|^{2}= \begin{cases}O(n) & \text { при } n / N \rightarrow 0, \\ O(n(1-n /(m N))) & \text { при } 0<a_{1} \leqslant n / N \leqslant a_{2}<\infty \\ O\left((1-n /(m N)) n^{3} / N^{2}\right) & \text { при } n / N \rightarrow \infty\end{cases}
$$

Отсюда и (15) следует, что при выполнении условий леммы справедливо соотношение

$$
\left\|Q_{N}^{-1}\right\|^{2}=O\left(H_{N}\right)
$$

Лемма доказана.

Аналогично леммам 3, 4 доказываются следующие утверждения.

Лемма 5. Пусть $\xi_{1}, \ldots, \xi_{N}$ имеют распределение (4), где

$$
x=\frac{n}{n+N} \exp \left\{-\frac{n}{n+N}\right\},
$$

$n, N \rightarrow \infty$ так, что $n^{2} / N \rightarrow \infty, n / N^{2} \rightarrow 0$.

Тогда последовательность распределений $\left(S_{N}-A_{N}\right) Q_{N}$ слабо сходится к двумерному нормальному закону с плотностью (9). 
Лемма 6. Пусть $\xi_{1}, \ldots, \xi_{N}$ имеют распределение (4), где х определяется соотночением (16), $n, N \rightarrow \infty$ так, что $n^{2} / N \rightarrow \infty, n / N=O(1)$. Тогдa

$$
\mathbf{P}\left\{S_{n}=z\right\}=\operatorname{det} Q_{N}\left(g\left(\left(z-A_{N}\right) Q_{N}\right)+o(1)\right)
$$

равномерно по $z=(k, l)$, где $k, l$ - челые неотрицательные числа.

Лемма 7. Пусть $\xi_{1}, \ldots, \xi_{N}$ имеют распределение (5), где $\lambda$ - решение уравнения (6) в интервале $(0,1), n>N, n, N \rightarrow \infty$ так, что $(n-N)^{3} / N^{2} \rightarrow \infty, N / \ln n \rightarrow \infty$.

Тогда последовательность распределений $\left(S_{N}-A_{N}\right) Q_{N}$ слабо сходится к двумерному нормальному закону с плотностью (9).

Лемма 8. Пусть $\xi_{1}, \ldots, \xi_{N}$ имеют распределение (5), где $\lambda$-решение уравнения (6) в интервале $(0,1), n, N \rightarrow \infty$ так, что $1<a_{1} \leqslant n / N \leqslant a_{2}<\infty$. Тогда

$$
\mathbf{P}\left\{S_{n}=z\right\}=\operatorname{det} Q_{N}\left(g\left(\left(z-A_{N}\right) Q_{N}\right)+o(1)\right)
$$

равномерно по $z=(k, l)$, где $k=0,1, \ldots, l=N, N+1, \ldots$

Теперь перейдем к доказательству теорем 1-3.

Пусть $\xi_{1}, \ldots, \xi_{N}$ имеют биномиальное распределение с параметрами $m \geqslant 2$ и $p=n /(m N)$. Следуя доказательству теоремы Муавра-Лапласа, можно показать, что при $n, N \rightarrow \infty, m N-n \rightarrow \infty$ имеет место соотношение

$$
\mathbf{P}\left\{\mu_{N}=k\right\}=\frac{1}{\sigma_{2} \sqrt{2 \pi N}} \exp \left\{-\frac{(k-n)^{2}}{2 \sigma_{2}^{2} N}\right\}(1+o(1))
$$

равномерно по всем $k$, для которых $(k-n) / \sigma_{2} \sqrt{N}$ лежит в любом фиксированном конечном интервале. Утверждение теоремы 1 вытекает из (3), (17) и леммы 4.

Пусть $\xi_{1}, \ldots, \xi_{N}$ имеют распределение (4), где $x$ определяется соотношением (16). В [7] доказано, что

$$
\mathbf{P}\left\{\mu_{N}=n\right\}=\frac{N}{(n+N) \sqrt{2 \pi n}}(1+o(1)) .
$$

Утверждение теоремы 2 следует из последнего равенства, (3) и леммы 6.

Пусть $\xi_{1}, \ldots, \xi_{N}$ имеют распределение (5). Согласно теореме 4.2 .7 из [5] имеет место соотношение

$$
\mathbf{P}\left\{\mu_{N}=n\right\}=\frac{1}{\sigma_{2} \sqrt{2 \pi N}}(1+o(1)),
$$

из которого, а также (3) и леммы 8 следует утверждение теоремы 3. 


\section{Список литературы}

1. Колчин В. Ф., О распределении одной статистики в полиномиальной схеме. Труды Московского института электронного машиностроения (1973) 32, 73-91.

2. Агиевич С. В., Об одной комбинаторной задаче о размещениях. Компьютерный анализ данных и моделирование. Сб. научных статей V международной конференчии, ч.3, Минск, 1998, 28-31.

3. Колчин В. Ф., Севастьянов Б. А., Чистяков В. П., Случайные размещения. Наука, Москва, 1976.

4. Колчин В. Ф., Случайные отображения. Наука, Москва, 1984.

5. Колчин В. Ф., Случайные графы. Физматлит, Москва, 2000.

6. Pavlov Yu. L., Random forests. VSP, Utrecht, 2000.

7. Павлов Ю. Л., Асимптотическое распределение максимального объема дерева в случайном лесе. Теория вероятностей и ее применения (1977) 22, №3, 523-533.

8. Мухин А. Б., Локальные предельные теоремы для решетчатых случайных величин. Теория вероятностей и ее применения (1991) 36, №4, 660-674.

Статья поступила 17.06.2002. 